\title{
Pascal Rannou, De Corbière à Tristan. Les Amours jaunes: une quête de l'identité
}

\section{Mario Richter}

\section{Q OpenEdition}

1 Journals

\section{Edizione digitale}

URL: http://journals.openedition.org/studifrancesi/9627

DOI: 10.4000/studifrancesi.9627

ISSN: 2421-5856

\section{Editore}

Rosenberg \& Sellier

\section{Edizione cartacea}

Data di pubblicazione: 1 décembre 2007

Paginazione: 688

ISSN: 0039-2944

\section{Notizia bibliografica digitale}

Mario Richter, «Pascal Rannou, De Corbière à Tristan. Les Amours jaunes: une quête de l'identité», Studi Francesi [Online], 153 (LI | III) | 2007, online dal 30 novembre 2015, consultato il 13 janvier 2021. URL: http://journals.openedition.org/studifrancesi/9627 ; DOI: https://doi.org/10.4000/studifrancesi.9627

Questo documento è stato generato automaticamente il 13 janvier 2021.

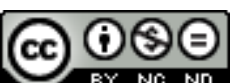

Studi Francesi è distribuita con Licenza Creative Commons Attribuzione - Non commerciale - Non opere derivate 4.0 Internazionale. 


\title{
Pascal Rannou, De Corbière à Tristan. Les Amours jaunes: une quête de l'identité
}

\author{
Mario Richter
}

\section{NOTIZIA}

PASCAL RANNOU, De Corbière à Tristan. Les Amours jaunes: une quête de l'identité, Paris

Champion, 2006, pp. 543.

1 Il presente libro si raccomanda alla lettura perché costituisce finora il più organico $\mathrm{e}$ persuasivo tentativo di approfondimento dell'opera poetica di Corbière considerata nella sua coesione e nella sua coerenza grazie all'attenta analisi di un gran numero di testi. Il metodo adottato dall'A., che egli stesso definisce etno-stilistico, si avvale soprattutto dei lavori di Sélim Abou (se ne veda, in particolare, L'Identité culturelle, 1989). Dopo aver dedicato un ampio e utile capitolo introduttivo alle opere critiche dedicate alle Amours jaunes, Rannou prende le mosse dalla verifica di quella che egli chiama "identité contrariée", ossia il rifiuto da parte di Corbière delle risorse linguistiche che costituiscono il più solido supporto dei valori che gli sono stati impartiti dall'ambiente della sua iniziale formazione umana e culturale. La seconda parte, dedicata all'“identité niée", mostra in quale modo Corbière è riuscito a fare tavola rasa di tutto il complesso di condizionamenti mentali di cui si è trovato a essere l'erede. La terza parte studia il momento più drammatico, quello del "désarroi identitaire", in cui lo sradicamento e l'esilio assumono, in testi spiccatamente nichilisti, il significato di principi creatori. A questo punto si evidenzia in Corbière la volontà di tornare verso un'età dell'oro familiare o etnica. Nella quarta parte, intitolata "l'identité retrouvée" il protagonista delle Amours jaunes si ricompone aderendo ai gruppi culturali che gli erano stati perentoriamente negati dalla sua iniziale educazione. Questo piano di studio potrebbe far pensare a un certo schematismo. In realtà l'A. è particolarmente sensibile alla realtà del testo, che non consente facili semplificazioni. Il 
suo impegno consiste nel cogliere la natura effettiva e complessa, esaltante e dolorosa, della "quête identitaire", ossia della progressiva ridefinizione del personaggio che assicura l'unità delle Amours jaunes (costruite e organiche come lo sono, a una attenta lettura, Les Fleurs du Mal di Baudelaire, a cui Corbière guarda con particolare interesse): l'A. si impegna a mostrare la difficile strada umana e letteraria che ha dovuto percorrere Edouard-Joachim Corbière per diventare Tristan. Quest'ultimo, staccandosi dai valori normativi della famiglia di Edouard-Joachim, avrebbe trovato la sua identità votandosi a tre realtà marginali, a quelle dei marinai, della bohème parigina e dei "Bretons bretonnants". «Se nourrissant d'apports ethniques et artistiques nouveaux scrive l'A. - la culture de Corbière se montre mobile, diverse, polyphonique», arrivando in tal modo ad accostarla a tendenze attuali della letteratura del Québec, del Maghreb, delle Antille...

Il libro si conclude con una bibliografia particolarmente ricca. 